\title{
Hierarchical Structure in Financial Markets
}

\author{
Rosario N. Mantegna \\ Istituto Nazionale per la Fisica della Materia, Unità di Palermo \\ and \\ Dipartimento di Energetica ed Applicazioni di Fisica, Università di Palermo, Viale delle Scienze, \\ I-90128, Palermo, Italy
}

\begin{abstract}
I find a topological arrangement of stocks traded in a financial market which has associated a meaningful economic taxonomy. The topological space is a graph connecting the stocks of the portfolio analyzed. The graph is obtained starting from the matrix of correlation coefficient computed between all pairs of stocks of the portfolio by considering the synchronous time evolution of the difference of the logarithm of daily stock price. The hierarchical tree of the subdominant ultrametric space associated with the graph provides information useful to investigate the number and nature of the common economic factors affecting the time evolution of logarithm of price of well defined groups of stocks.
\end{abstract}

Financial markets are well defined complex systems. They are studied by economists, mathematicians and, recently, also by physicists. The paradigm of mathematical finance is that the time series of stock returns are unpredictable [1]. Within this paradigm, time evolution of stock returns are well described by random processes. A key point is if the random processes of stock returns time series of different stocks are uncorrelated or, conversely, if common economic factors are present in financial markets and are driving several stocks at the same time. Common economic factors were originally introduced by Ross in 
his arbitrage pricing theory model [2]. Since its introduction, the problem of the number and nature of common economic factors has been a considerable controversial issue. On the side of modeling of financial markets by using tools and procedures developed to model physical systems [3 [10], there is the need to characterize the topological arrangement of different stocks traded in a financial markets. Similar information is essential to attempt to model financial markets in terms of nonlinear systems in the presence of external and/or quenched noise.

The motivation of the present study is twofold. The first motivation concerns the search for the kind of topological arrangement which is present between the stocks of a portfolio traded in a financial market. The second motivation is the search of empirical evidence about the existence and nature of common economic factors which drive the time evolution of stock prices.

In the present analysis, I investigate the hierarchical structure present in a portfolio of $n$ stocks traded in a financial market. The observable which is used to detect the topological arrangement of the stocks of a given portfolio is the synchronous correlation coefficient of the daily difference of logarithm of closure price of stocks. The correlation coefficient is computed between all the possible pairs of stocks present in the portfolio in a given time period. In this letter, I report results obtained by investigating the portfolio of the stocks used to compute the Dow Jones industrial average index and the portfolio of stocks used to compute the Standard and Poor's 500 index in the time period from July 1989 to October 1995. Both indices mainly describe the performance of the New York Stock Exchange.

The starting point of my investigation is to quantify the degree of similarity between the synchronous time evolution of a pair of stock price by the correlation coefficient [11]

$$
\rho_{i j}=\frac{<Y_{i} Y_{j}>-<Y_{i}><Y_{j}>}{\sqrt{\left(<Y_{i}^{2}>-<Y_{i}>^{2}\right)\left(<Y_{j}^{2}>-<Y_{j}>^{2}\right)}}
$$

where $i$ and $j$ are the numerical labels of stocks, $Y_{i}=\ln P_{i}(t)-\ln P_{i}(t-1)$ and $P_{i}(t)$ is the closure price of the stock $i$ at the day $t$. The statistical average is a temporal average performed on all the trading days of the investigated time period. 
For both portfolios, I determine the $n \times n$ matrix of correlation coefficients for daily logarithm price differences (which almost coincides with returns). By definition, $\rho_{i j}$ can vary from -1 (completely anti-correlated pair of stocks) to 1 (completely correlated pair of stocks). When $\rho_{i j}=0$ the two stocks are uncorrelated.

The matrix of correlation coefficients is a symmetric matrix with $\rho_{i i}=1$ in the main diagonal. Hence, in each portfolio, $n(n-1) / 2$ correlation coefficients characterize the matrix completely. An investigation of the statistical properties of the set of correlation coefficients is published elsewhere [12]. In this letter, I investigate the correlation coefficient matrix to detect the hierarchical organization present inside the stock market. In the search for an appropriate topological arrangement of stocks of a given portfolio, I first look for a metric. The correlation coefficient of a pair of stocks cannot be used as a distance between the two stocks because it does not fulfill the three axioms that define an Euclidean metric. However a generalized metric can be defined using as distance an appropriate function of the correlation coefficient. The chosen function is

$$
d(i, j)=1-\rho_{i j}^{2} .
$$

With this choice $d(i, j)$ fulfills the three axioms of an Euclidean metric - (i) $d(i, j)=0$ if and only if $i=j$; (ii) $d(i, j)=d(j, i)$ and (iii) $d(i, j) \leq d(i, k)+d(k, j)$, for all practical purposes. The first axiom is valid because $d(i, j)=0$ if and only if the correlation (or the anticorrelation) is complete $(|\rho|=1$, namely only if the two stocks perform the same stochastic process or stochastic processes which are mirror images the one of the other). The second axiom is valid because the correlation coefficient matrix and hence the distance matrix $\mathbf{D}$ is symmetric by definition, and the third axiom has been verified numerically in all portfolios investigated.

The distance matrix $\mathbf{D}$ is then used to determine the minimal spanning tree [13] connecting the $n$ stocks of the portfolio. The minimal spanning tree (MST) is attractive because provides an arrangement of stocks which selects the most relevant connections of each point of the set. Moreover the minimal spanning tree gives, in a direct way, the subdominant ul- 
trametric hierarchical organization of the points (stocks) of the investigated portfolio. In the rest of this letter, I will show that the hierarchical organization found through the minimal spanning tree associated with the distance matrix $\mathbf{D}$ is of great interest from an economic point of view. In particular, by assuming this kind of topology, I am able to isolate groups of stocks which makes sense from an economic point of view by starting from the information carried by the time series of prices only. The classification of the groups of stocks obtained with my analysis of the correlation coefficients is performed by using the industry and subindustry sectors reported in the Forbes 49th annual report on American industry.

For each portfolio investigated, starting from the distance matrix $\mathbf{D}$, it is possible to obtain the minimal spanning tree (MST) connecting the stocks of the portfolio. In fig. 1a, I show the minimal spanning tree for the Dow Jones industrial average portfolio of stocks. Each circle represents a stock, labeled by its tic symbol (for example KO is Coca Cola Com. , PG is Procter \& Gamble, etc. See the caption of the figure for more details). The connections between stocks are shown by segments and the color of these segments is related to the distance between stocks. In fig. 1b the hierarchical tree of the subdominant ultrametric 114 associated to the MST is shown. An inspection of the MST and of the associated hierarchical tree show the existence of three groups of stocks. The observed grouping has a direct economic explanation. The more evident and strongly connected group is the group of stocks CHV, TX and XON namely Chevron, Texaco and Exxon. These three companies are working in the same industry (energy) and in the same subindustry (international oils). A second group is formed by AA and IP, namely by Alcoa (working in the subindustry sector of nonferrous metals) and International Paper (working in the subindustry sector of paper and lumber). Both companies provide raw materials. The third group involves companies which are in industry sectors which deals with consumer nondurables (Procter \& Gamble, PG) and food drink and tobacco (Coca Cola, KO).

The same investigation is repeated for the set of stocks used to compute the Standard and Poor's 500 index. In this case the larger size of the portfolio allows to perform a more refined test of the detected hierarchical structure of stocks. In my analysis, I considered only 
the companies which were present in the S\&P 500 index for the entire period investigated. With this constrain the portfolio is composed of 443 stocks. Due to the size of the portfolio investigated, the obtained minimal spanning tree cannot be shown in a single figure in a legible way. In Fig. 2, I show some of the parts of the MST which are strongly connected. A group of financial services, capital goods, retailing, food drink \& tobacco and consumer nondurables companies is shown in I; the group of oil companies is shown in II, while III is the group of communication and electrical utility companies and IV is the group of raw material companies. The portfolio of stocks used to compute the S\&P 500 index is characterized by a hierarchical structure of stocks which is much more detailed than the one observed in the case of the DJIA portfolio. The structure of the minimal spanning tree of the portfolio of stocks of the S\&P 500 index, shows many groups of stocks which are homogeneous from an economic point of view. A detailed inspection of the hierarchical tree associated to the MST provides a large amount of economic information. It is impossible to put in a single legible figure the complete hierarchical tree of a so broad portfolio. In fig. 3, we then show only the branching of the tree up to the level of homogeneous groups. This means that lines in the hierarchical tree shown in fig. 3 are always ending in a group of stocks which contains at least 2 stocks (but usually more). The branches of single stocks departing from the tree are not shown to make the figure readable. In the caption of fig. 3, I give full details about the stocks belonging to the groups shown in the figure, together with their classification in industry sectors and/or subsectors. With only a few exceptions the groups are homogeneous with respect to industry and often also subindustry sectors suggesting that set of stocks working in the same industry and subindustry sectors respond, in a statistical way, to the same economic common factors. In some cases, my analysis, based on the statistical analysis of correlation coefficients between pairs of stock returns, refines the classification in sectors and subsectors used by Forbes. For example, ores, aluminum and copper are all classified metals as industry and nonferrous metals as subindustry. From my analysis, I detect that they respond to quite different common economic factors. Specifically, ores companies are grouped in a cluster which is the most distant form all the others groups 
of stocks of the tree, while aluminum and copper companies constitute a subgroup of the group containing raw materials companies.

The detection of a hierarchical structure in a broad portfolio of stocks traded in a financial market is consistent with the assumption that the time series of returns of a stock is affected by a number of economic factors. The analysis shows that the number and the relative influence of these factors is specific to each stock. In general, stocks or groups of stocks that depart early from the tree (at high values of the distance $d(i, j))$ are mainly controlled by economic factors which are specific to the considered group (for example gold price for the stocks of the group 1 of the tree (see Fig. 3) which is composed only by companies involved in gold mining). When departure occurs for (moderately) low values of $d$, the stocks are affected either by economic factors which are common to all stocks and by other economic factors which are specific to the considered set of stocks. The relative relevance of these factors is quantified by the length of the segment (or segments) observed for each group from one branching to the successive one.

In conclusion, the present study shows that the MST and the associated subdominant ultrametric hierarchical tree, obtained starting from the distance matrix of Eq. (2), selects a topological space for the stocks of a portfolio traded in a financial market which is able to give an economic meaningful taxonomy. This topology is useful in the theoretical description of financial markets and in the search of economic common factors affecting specific groups of stocks. The topology and the hierarchical structure associated to it, is obtained by using information present in the time series of stock prices only. This result shows that time series of stock prices are carrying valuable (and detectable) economic information.

\section{ACKNOWLEDGMENTS}

I thank INFM and MURST for financial support. 


\section{REFERENCES}

[1] Samuelson, P. A., Industrial Management Review Vol. 6, No. 2 , 41-49 (1965); reproduced as Chapter 198 in Samuelson, Collected Scientific Papers, Volume III, Cambridge, M.I.T. Press, 1972.

[2] Ross, S. A., Journal of Economic Theory 13, 341-360 (1976).

[3] Mandelbrot, B. B., J. Business 36, 394-419 (1963).

[4] Kadanoff, L. P., Simulation 16, 261 (1971).

[5] Mantegna, R. N., Physica A 179, 232-242 (1991).

[6] Bak, P., Chen, K., Scheinkman, J. A., and Woodford, M., Ricerche Economiche 47, 3-30 (1993).

[7] Bouchaud, J.-P. and Sornette, D., J. Phys. I France 4, 863-881 (1994).

[8] Mantegna, R. N., and Stanley, H. E., Nature 376, 46-49 (1995).

[9] Ghashghaie, S., Breymann, W., Peinke, J., Talkner, P., and Dodge, Y., Nature 381, 767-769 (1996).

[10] Bak, P., Paczuski, M., and Shubik, M., Physica A 246, 430-453 (1997).

[11] Feller W., An Introduction to Probability Theory and Its Applications (Wiley, New York) 1971.

[12] Mantegna, R. N., "Degree of Correlation Inside a Financial Market" in [Proc. of the ANDM 97 International Conference], Edited by J. Kadtke, AIP press, (1997).

[13] Papadimitriou, C. H., and Steiglitz, K., Combinatorial Optimization (Prentice-Hall, Englewood Cliffs, 1982).

[14] Rammal, R., Toulouse, G., and Virasoro, M. A., Rev. Mod. Phys. 58, 765-788 (1986). 


\section{FIGURES}

FIG. 1. (a) Minimal spanning tree connecting the 30 stocks used to compute the Dow Jones Industrial Average. The distance matrix $\mathbf{D}$ is obtained starting from the correlation coefficients measured between all pairs of stocks in the portfolio in the time period from July 89 to October 95. The 30 stocks are labeled by their tic symbols (AA-Alcoa, ALD-Allied Signal, AXP-American Express Co, BA-Boeing Co, BS-Bethlehem Steel, CAT-Caterpillar Inc., CHV-Chevron Corp., DD-Du Pont, DIS-Walt Disney Co., EK-Eastman Kodak Co., GE-General Electric, GM-General Motors, GT-Goodyear Tire, IBM-IBM Corp., IP-International Paper, JPM-Morgan JP, KO-Coca Cola Co., MCD-McDonaldss Corp., MMM-Minnesota Mining, MO-Philips Morris, MRK-Merck \& Co Inc., PG-Procter \& Gamble, S-Sears Roebuck, T-AT\&T, TX-Texaco Inc., UK-Union Carbide, UTX-United Tech, WX-Westinghouse, XON-Exxon Corp. and Z-Woolworth). The colors of segments connecting stocks are proportional to the distance between the stocks (yellow $0.65<d(i, j) \leq 0.70$, green $0.70<d(i, j) \leq 0.75$, turquoise $0.75<d(i, j) \leq 0.80$, cyan $0.80<d(i, j) \leq 0.85$, blue $0.85<d(i, j) \leq 0.90$ and violet $0.90<d(i, j) \leq 0.95)$. (b) Hierarchical tree of the subdominant ultrametric associated with the minimal spanning tree of a). In the tree, groups of stocks, homogeneous with respect to the economic activities of the companies are detected: (i) oil companies (Exxon, Texaco and Chevron); (ii) raw material companies (Alcoa and International paper) and (iii) companies working in the sectors of consumer nondurable products (Procter \& Gamble) and food and drinks (Coca Cola). The distance at which individual stocks are branching from the tree is given by the $y$ axis. 
FIG. 2. Partial regions of the minimal spanning tree of the portfolio of stocks used to compute the S\&P 500 index. The distance matrix $\mathbf{D}$ is obtained starting from the correlation coefficients measured between all pairs of stocks in the portfolio traded in the time period from July 89 to October 95. The four panels show four strongly connected large groups of stocks observed for $d(i, j) \leq 0.81$. Circles represents stocks which are labeled by their stock exchange tic symbols. The colors of segments joining circles are proportional to the distance between stocks (magenta $0.5<d(i, j) \leq 0.55$, red $0.55<d(i, j) \leq 0.6$, orange $0.6<d(i, j) \leq 0.65$ and other colors as in fig. 1a). The dashed segments are connections between stocks which join different regions of the minimal spanning tree having distances $d(i, j)>0.81$. In panel I, the group of financial service companies (AHM, BAC, BBI, BK, BKB, BT, CCI, CHL, CMB, FFB, FNB, FNM, FTU, GDW, GWF, I, JPM, MER, ONE, PNC, SNC and WFC), capital goods companies (EMR and GE), retailing companies (HD and WMT), consumer nondurables companies (CL and PG) and food and drinks companies (KO and PEP) are shown. Du Pont company (DD) is joining this group of stocks to the group of oil companies of panel II. Panel II shows international oil companies (AN, CHV, MOB, RD, TX and XON), other energy companies (AHC, ARC, KMG, P and UCL) and oilfield service companies (BHI, DI, HAL and SLB). UN (Unilever) is the only stock of the group which is not directly homogeneous to industry subsectors of international oil and other energy. Panel III shows companies which are working in the industry sector of electric utilities (AEP, BGE, CPL, CSR, D, DTE, DUK, ED, FPL, NSP, OEC, PCG, PE, PEG and SO) and in the subindustry sector of telecommunications (AIT, BEL, BLS, GTE, NYN, SBC and USW). T, namely AT\&T Corp., connects this group of stocks to other groups through General Electric (GE). Last panel (IV) shows a group of companies with activities in the sectors of raw materials. Two subgroups are observed: (i) companies working in the industry sector of metals and subindustry nonferrous materials (AA, AL, AR, PD, N and RLM) and companies working in the industry sector of forest products and packaging (CHA, IP, LPX, MEA, TIN, UCC and WY). The two subgroups are connected between them through the link between Alcoa (AA) and International paper (IP). They are connected to other groups through Minn Mining \& MFG (MMM) and General Electric. 
Fig. 3 Main structure of the minimal spanning tree of the portfolio of stocks used to compute the S\&P 500 index. Each line ending in the bottom corresponds to a group of stocks composed by at least two stocks. Lines are ending when the first bifurcation inside the group is observed. Individual stocks departing from the main tree are not shown for the sake of clarity. Groups are labeled with integers ranging from 1 to 44. The branching of each group from the main tree and inside the group are occurring at a distance given by the $d(i, j)$ scale. Below, I report for each group detected in the MST the observed common industry sector (IS) and subindustry sector (SS) together with the tic symbols of the stocks belonging to the group. The IS and SS of stocks are the ones used in the 49th Forbes annual report of American industry (accessible on the web at the address www.forbes.com). 1. IS metals, SS nonferrous metals (gold), stocks ABX, ECO, HM, NEM and PDG; 2. IS construction, SS residential builders, stocks CTX, KBH and PHM; 3. no common industry sector, stocks ACK and MAS; 4. IS travel and transport, SS trucking and shipping, stocks ROAD and YELL; 5. IS consumer nondurables, SS photography and toys, stocks HAS and MAT; 6. no common industry sector, stocks CBS and CCB; 7. IS metals, SS steel, stocks BS, IAD and X; 8. IS consumer durables, SS automotive parts, stocks DCN and ETN; 9. IS travel and transport, SS airlines, stocks AMR, DAL, LUV and U; 10. IS entertainment and information, SS broadcasting and cable, stocks CMCSA and TCOMA; 11. financial services, SS lease and finance, stocks BNL and HI; 12. IS energy, SS oilfield services, stocks BHI, DI, HAL and SLB; 13. IS energy, SS international oils, stocks AN, CHV, MOB, TX, XON and ARC (IS other energy); 14. no common industry sector, stocks RD and UN; 15. IS capital goods, SS heavy equipment, stocks CAT, DE, IR and TEN (IS forest products and packaging); 16. IS business services and supplies, SS environmental and waste, stocks BFI and WMX; 17. IS construction, SS commercial builders, stocks FLR and FWC; 18. IS consumer durables, SS automobiles and trucks, stocks C, F and GM; 19. IS food drink and tobacco, SS tobacco, stocks AMB and MO; 20. IS entertainment and information, SS publishing, stocks GCI, KRI and TRB; 21. IS forest products and packaging, SS paper and lumber, stocks BCC, CHA, IP, LPX, MEA, UCC, WY and TIN (SS packaging); 22. IS 
metals, SS nonferrous materials, stocks AR and PD; 23. IS metals, SS nonferrous materials, stocks AL and N; 24. IS metals, SS nonferrous materials, stocks AA and RLM; 25. IS computer and communications, SS peripherals \& equipment or software, stocks AMAT, CPQ, HWP, INTC, MOT, MSFT, NOVL, NSM and TXN; 26. IS Electric utilities, SS regional area, stocks AEP, BGE, CPL, CSR, D, DTE, DUK, ED, FPL, NSP, OEC, PCG, PE, PEG and SO; 27. IS computer and communications, SS telecommunications, stocks AIT, BEL, BLS, GTE, NYN, SBC and USW; 28. IS retailing, SS department stores and drug \& discount, stocks DH, DDS and MAY; 29. no common industry sector, stocks DD, DOW and VO; 30. IS travel and transport, SS railroads, stocks BNI, CRR, CSX, NSC and UNP; 31. IS food drink and tobacco, SS food processors, stocks CPC, GIS, K and SLE; 32. no common industry sector, stocks AET, and CI; 33. IS Insurance, SSs property \& casualty and diversified, stocks AIG, CB and GRN; 34. IS health, SS drugs, stocks PFE and SGP; 35. IS health, SS drugs, stocks BMY and MRK; 36. IS consumer nondurables, SS personal products, stocks CL and PG; 37. IS food drink and tobacco, SS beverages, stocks KO and PEP; 38. IS retailing, no common SS, stocks HD and WMT; 39. IS capital goods, SS electrical equipment, stocks EMR and GE; 40. IS financial services, no common SS, stocks FNM and GDW; 41. IS financial services, SS thrift institutions, stocks AHM and GWF; 42. IS financial services, SS multinational banks, stocks BT and JPM; 43. IS financial services, SS regional banks, stocks I (no more traded) and WFC; 44. IS financial services, SS multinational banks, stocks BAC, CCI, CHL (no more traded) and CMB. 


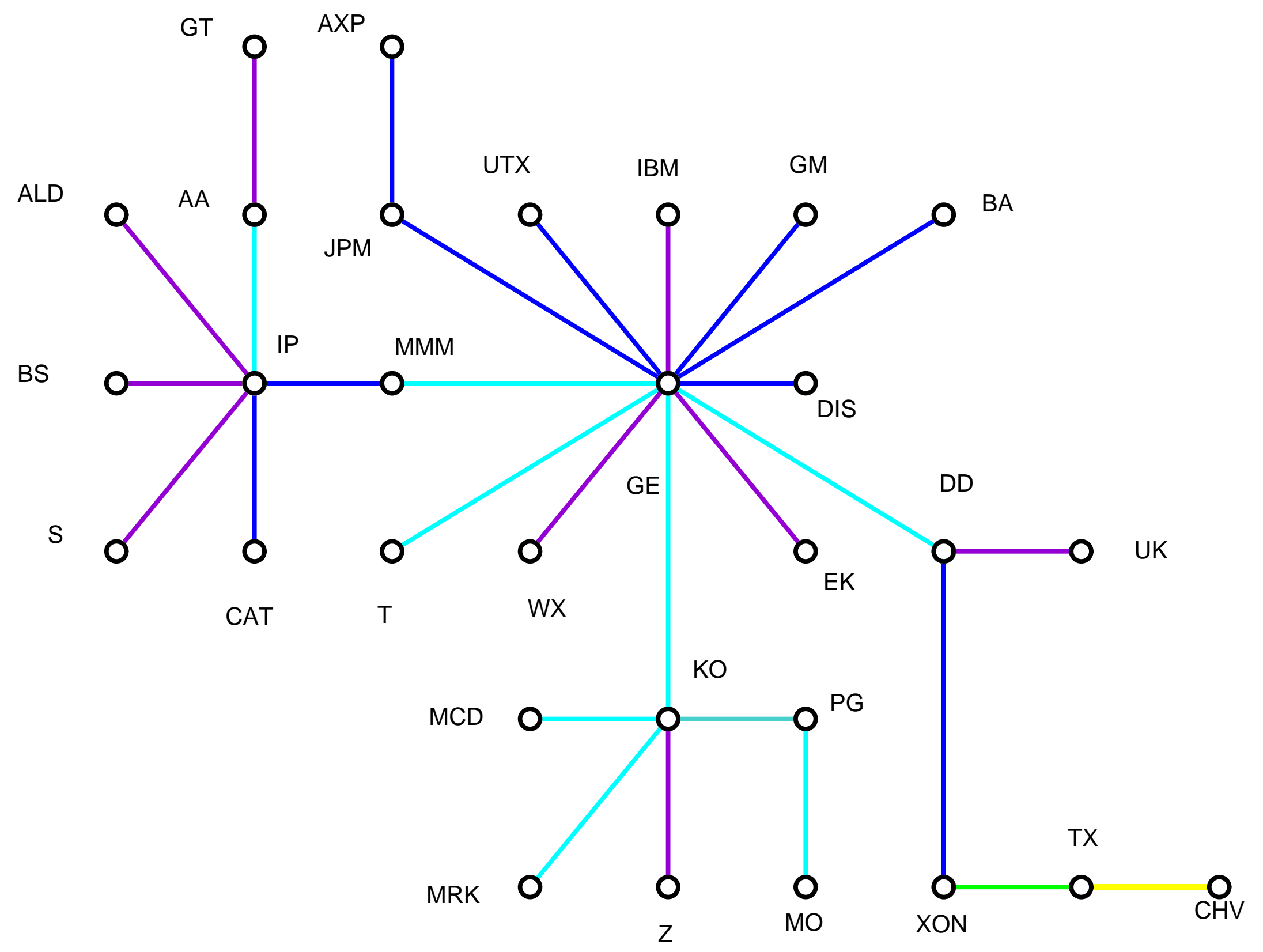




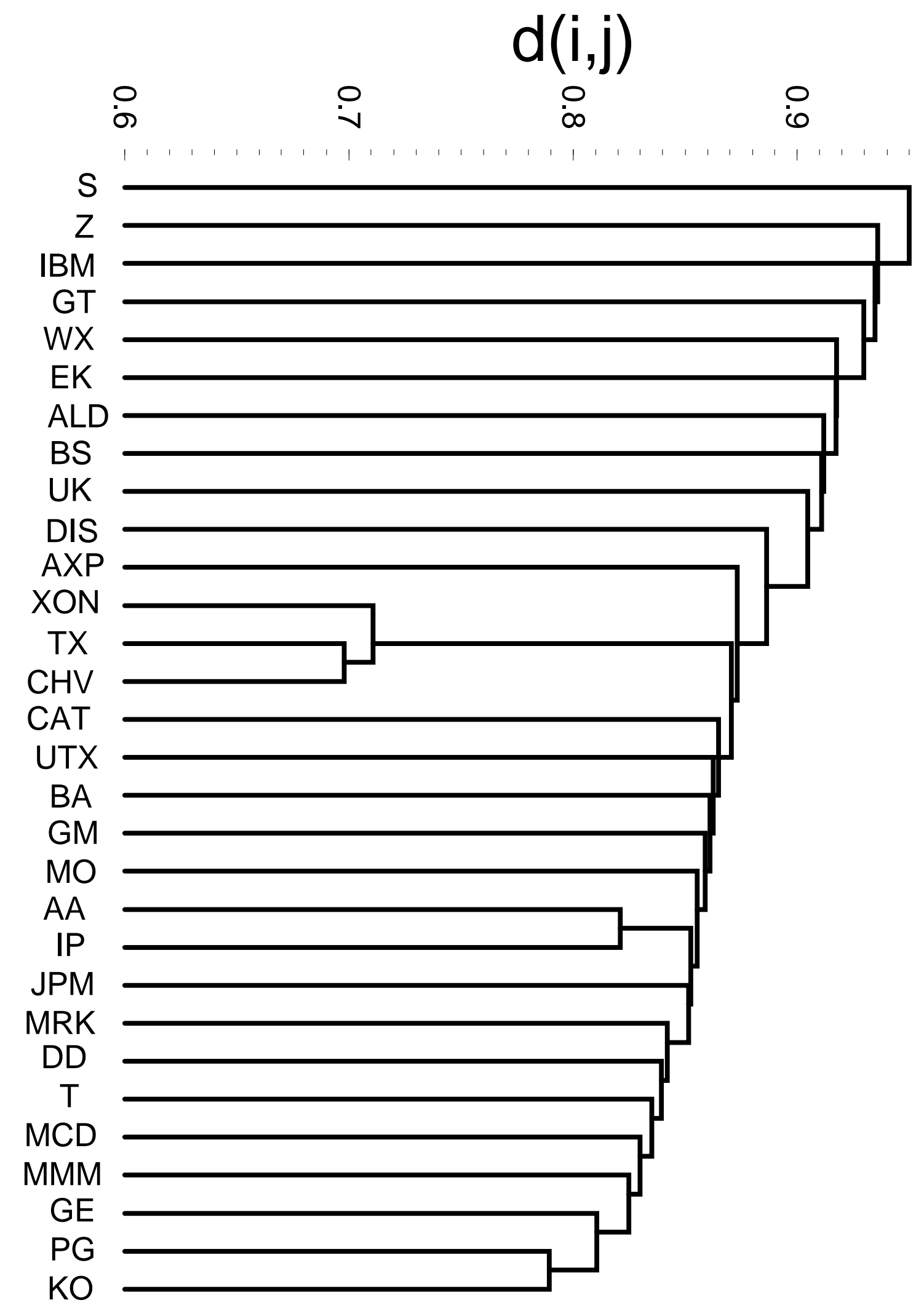




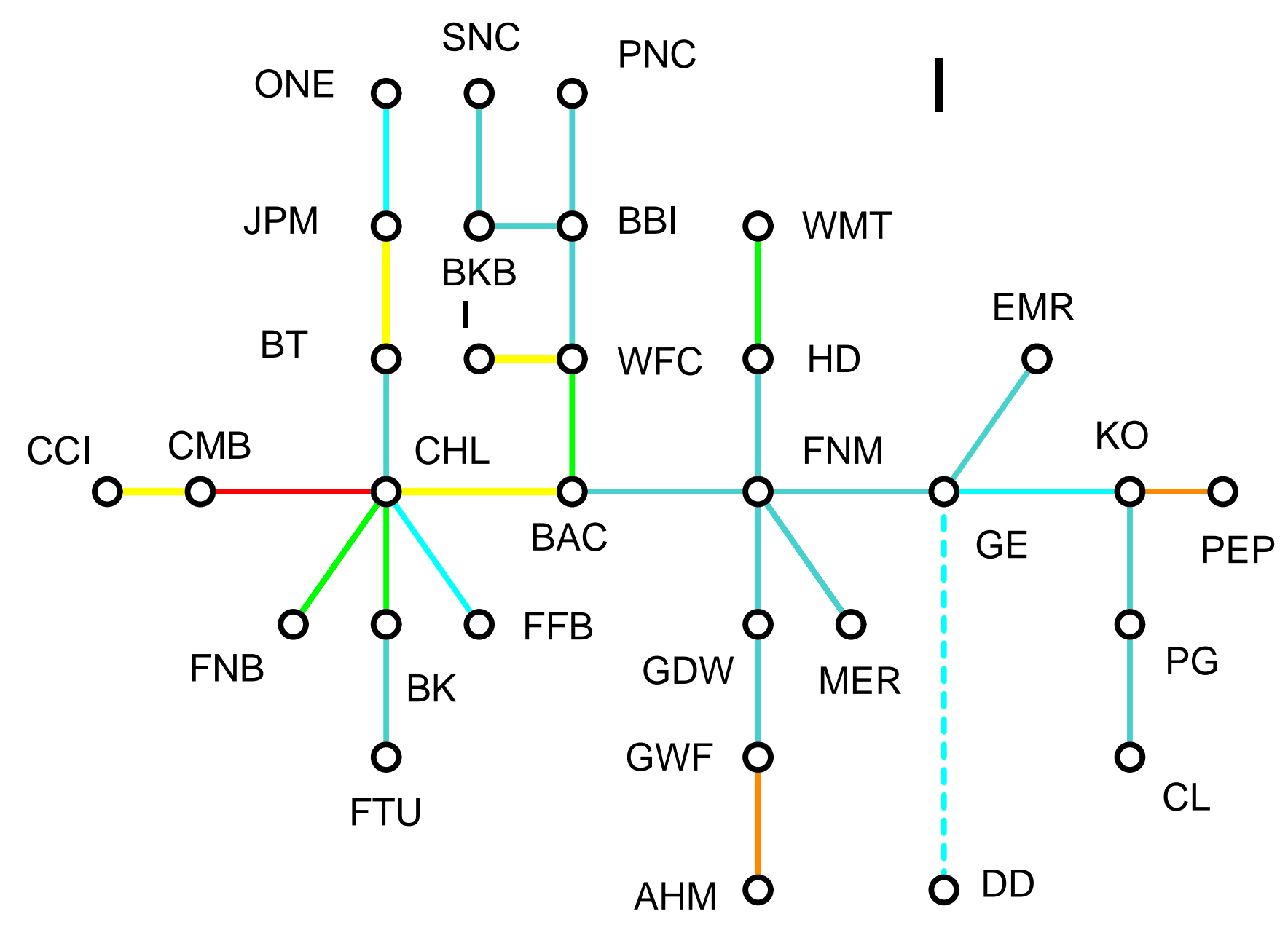




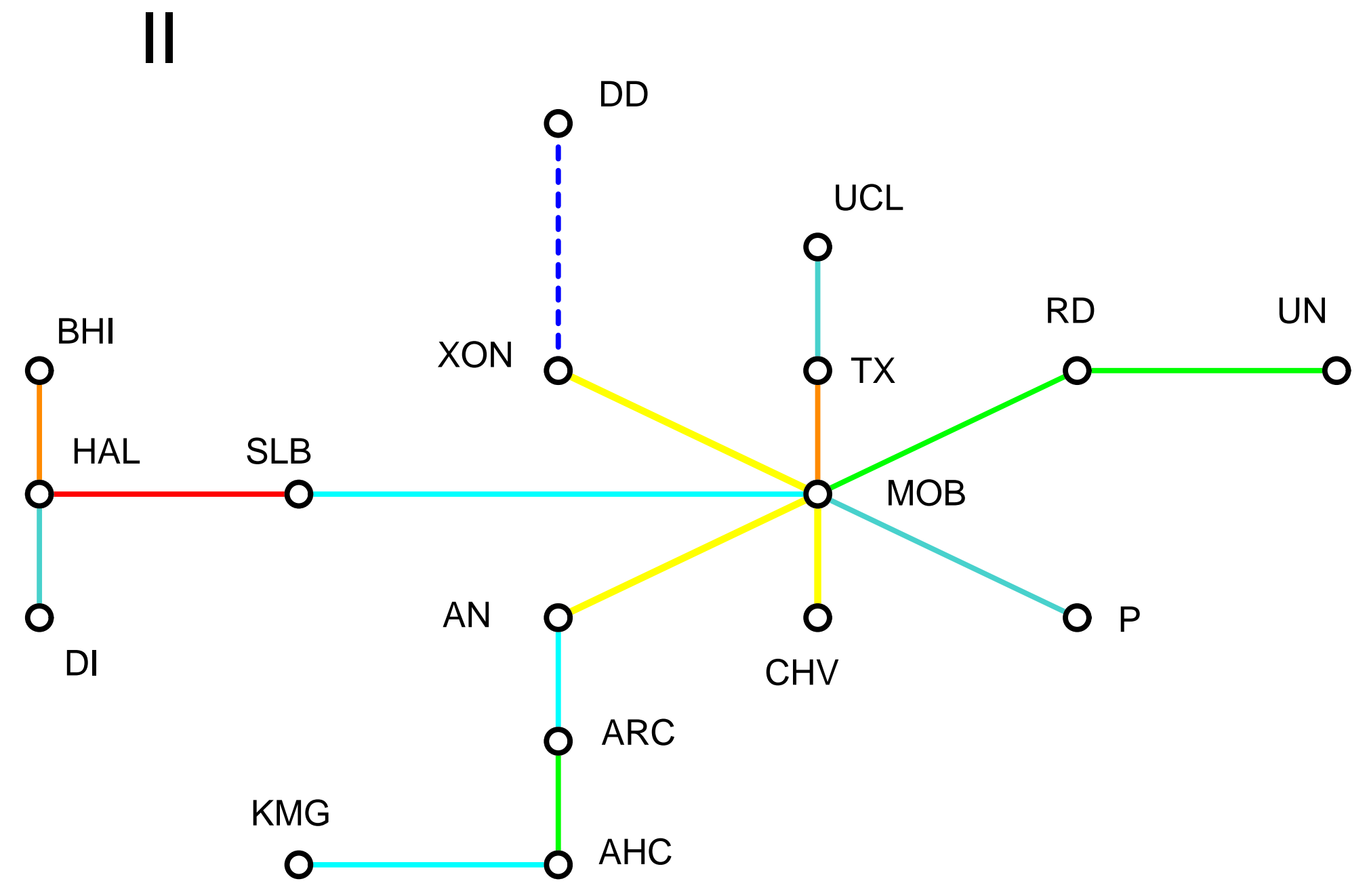




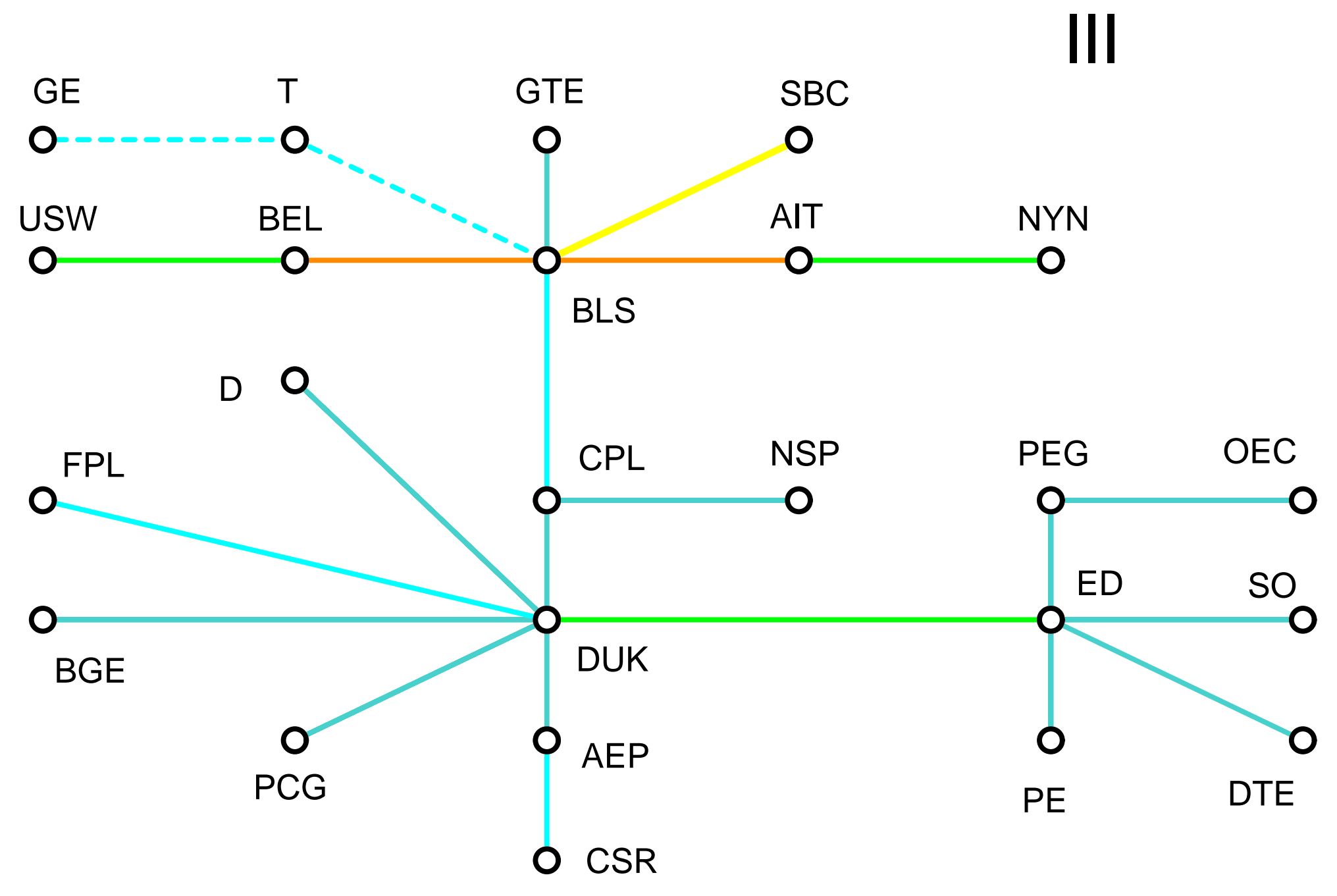




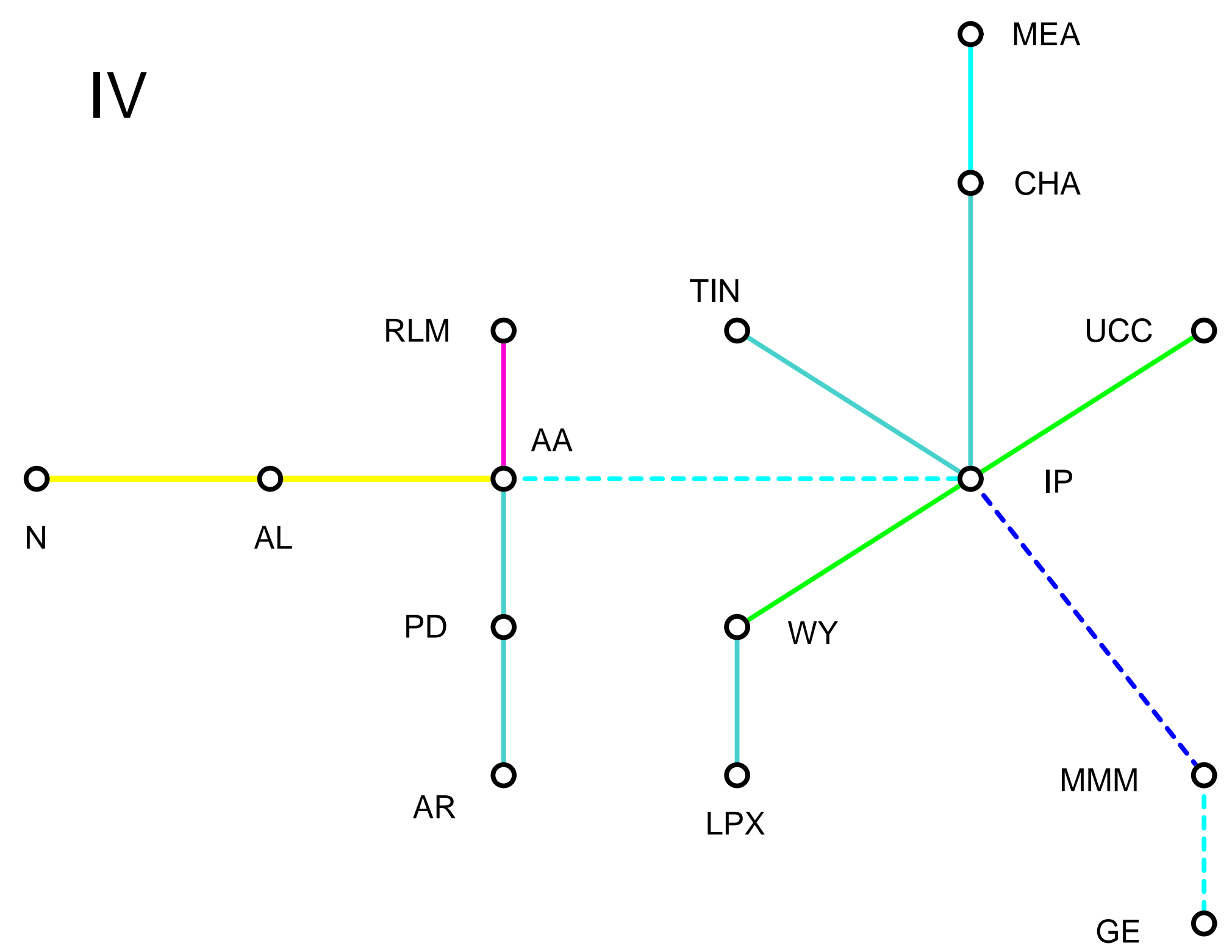




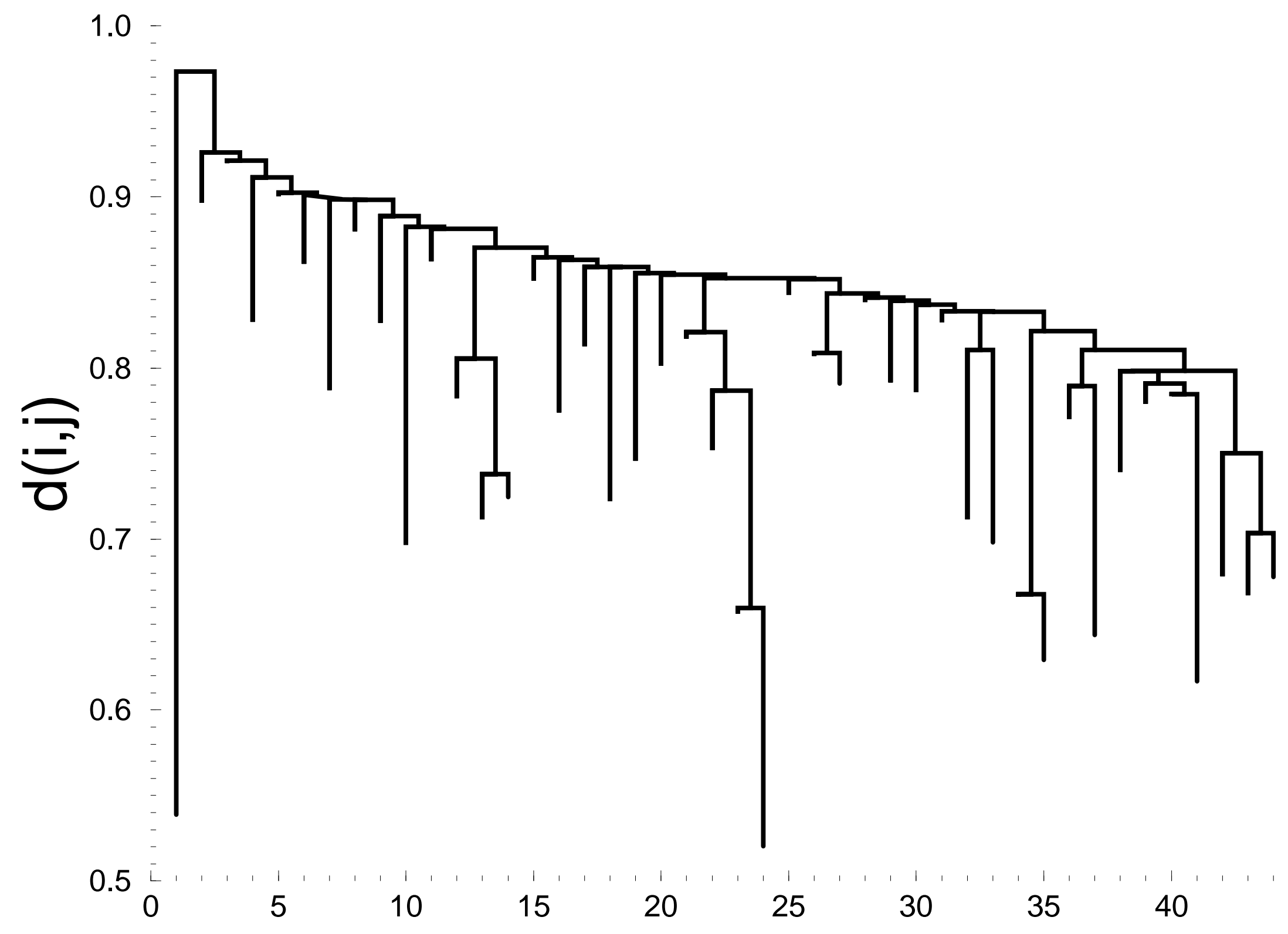

